\title{
On the need for comprehensive assessment of impact of comorbidity in elderly patients with head and neck cancer
}

\author{
Afshin Teymoortash • Gyorgy B. Halmos • \\ Carl E. Silver • Primož Strojan • Missak Haigentz Jr. • \\ Alessandra Rinaldo • Alfio Ferlito
}

Received: 17 June 2014 / Accepted: 17 July 2014/ Published online: 25 July 2014

(c) Springer-Verlag Berlin Heidelberg 2014

\begin{abstract}
Although a generally accepted definition of "elderly" still does not exist, in a medical context, persons over the age of 65 are often considered to be elderly. As life expectancy rates have increased within the last decades and continuously increase every year by approximately 3 months [1], this segment of the population has increased, particularly in Western countries. Because cancer is a disease of aging, the risk of developing malignant disease including head and neck cancer is highest in this patient population. It also
\end{abstract}

This paper was written by members and invitees of the International Head and Neck Scientific Group (http://www.IHNSG.com).

A. Teymoortash
Department of Otolaryngology-Head and Neck Surgery,
Philipp University, Marburg, Germany

G. B. Halmos

Department of Otorhinolaryngology-Head and Neck Surgery, University Medical Center Groningen, University of Groningen, Groningen, The Netherlands

\section{E. Silver}

Departments of Surgery and Otolaryngology-Head and Neck Surgery, Albert Einstein College of Medicine, Montefiore Medical Center, Bronx, NY, USA

P. Strojan

Department of Radiation Oncology, Institute of Oncology,

Ljubljana, Slovenia

M. Haigentz Jr.

Division of Oncology, Department of Medicine,

Montefiore Medical Center, Albert Einstein College of

Medicine, Bronx, NY, USA

A. Rinaldo $\cdot$ A. Ferlito $(\bowtie)$

University of Udine School of Medicine, Piazzale S. Maria della

Misericordia, 33100 Udine, Italy

e-mail: a.ferlito@uniud.it follows that malignancy-related mortality is highest among the elderly.

Younger patients with head and neck cancers have a much better overall prognosis. A comparative survival analysis revealed that patients with head and neck cancer who are aged 40 years or younger at the time of diagnosis show significantly improved 5-year survival when compared to older patients [2]. For all age groups the 5-year survival rate of patients with a newly diagnosed head and neck cancer is approximately $60 \%$ at diagnosis. However, prognosis is a dynamic process that improves after the initial critical years of recurrence-free survival. Conditional relative survival shows clinically relevant variations according to time since diagnosis, type of cancer, and age [3]. Conditional survival rates can better analyze and reflect actual life expectancy. After 3 years of survival, the conditional survival rate improves from $60 \%$ up to $80 \%$. However, long-term survival rates stagnate at $80 \%$. Patients cured of and neck cancer face an excess mortality of about $20 \%$ in long-term follow-up [4]. The critical reason for this is attributed to the comorbid burden of head and neck cancer patients, connected with unhealthy habits and general lifestyle choices.

Comorbidities in cancer patients are defined as one or more unrelated diseases present at the time of cancer diagnosis. Particularly in elderly patients, these predominantly consist of reduced organ and cognitive functions, malnutrition, polypharmacy, and socio-economic factors. Most studies show that approximately $60 \%$ of head and neck cancer patients have concurrent illnesses [5]. For better evaluation of comorbidities, weighted assessment systems like Adult Comorbidity Evaluation-27 (ACE-27), and Charlson Comorbidity Index (CCI) include the occurrence of comorbidities as well as their degrees. Comorbidities exist in $46 \%$ of head and neck cancer 
patients evaluated by CCI, with mild forms in $17 \%$ of cases, moderate in $9 \%$, and severe forms in $10 \%$ [6]. The existence of comorbidities increases with age. However, it declines beyond the age of 70 [7], suggesting that cancer survivors of this age have pursued a more healthy lifestyle or that those with high comorbid burden have already died.

Although most patients with head and neck cancer have curative-intent treatment plans with fair outcomes, the overall survival rate is influenced not only by recurrence but also by non-cancer causes. The overall survival rate declines with age because non-cancer related mortality is directly related to the severity of comorbidities. The cancer-specific mortality rate, however, remains independent of comorbidities [6]. This means that treatment outcomes are adjusted across different age groups for tumor stage, comorbidity burden and treatment intent [8].

As a result of comorbidities, patients who can potentially be cured may receive suboptimal treatment. Information on the disease itself and appropriate treatment modalities are often withheld during education of older patients who are often not sufficiently integrated into the decision making process regarding their treatment plan. Complex and expensive diagnostic tools may not be used, and multimodal therapy and extensive surgical procedures are often not applied. Denial of curative treatment to elderly patients may often produce an adverse effect by prolonging the patient's distress and increasing costs due to the need for long-term palliative care. Progression of suboptimally treated malignant disease can result in poorly controlled symptoms associated with persistent locoregional disease including dyspnea, dysphagia, tumor bleeding and ulcerations, and intractable pain. Abandonment of appropriate and standard curative treatment thus often leads to deterioration of prognosis and quality of life $[9,10]$.

The greatest challenge in the treatment of elderly head and neck cancer patients is the prediction of post-treatment outcome. It is known that the prediction of postoperative complications or radiotherapy-induced toxicity is much more difficult in the elderly than in the non-elderly population [11, 12]. Comorbidity as measured by different assessment systems does not seem to be sufficient to answer important questions such as expected post-treatment complications and the effects of cancer treatment on quality of life and survival. Such predictions would help in selecting appropriate elderly patients who might benefit from aggressive treatment.

Instead of analyzing only comorbid conditions, the comprehensive geriatric assessment (CGA) has been developed as a new holistic screening tool. The CGA includes, in addition to comorbidity evaluations, several other validated tools for evaluating functional, nutritional, cognitive and psychological status, social support, and physical performance [13]. The CGA has been found beneficial for predicting treatment-related complications as well as preventing hospitalization and geriatric syndromes, recognition of cognitive deficits, and improving survival $[14,15]$.

Functional status and physical performance can be evaluated by different activity of daily living (ADL) and instrumental ADL (IADL) tests. ADL evaluation focuses on the basic skills to maintain independent activities at home such as bathing, dressing, self feeding, and mobility. IADL tests assess ability to function independently in the community activities, such as shopping, transportation, and housework. CGA studies have demonstrated the predictive value of these ADL/IADL tests with regard to survival, postoperative morbidity and mortality, and chemotherapy toxicity [13]. As an alternative to time-consuming evaluations, some very simple measures have been developed to evaluate functional performance, such as the "Timed Up \& Go", which appears to be valuable for predicting postoperative complications after oncologic surgery [16]. Shortened forms of CGA have been proposed, although they require validation in routine clinical settings [17].

Nutritional state at the time of diagnosis of cancer as well as during the treatment process is of high importance. Several validated measurement tools are available for screening feeding status, such as the Nutritional Risk Screening 2002 questionnaire or the Malnutrition Screening Tool (MST) [18]. These tests help clinicians identify high-risk patients who would benefit from active nutritional support such as pre-treatment preventive nasogastric or percutaneous feeding tube placement, as was recently reported in a series of head and neck cancer patients [19].

The importance of evaluation of cognitive state is highlighted in the literature. At initial screening, up to $50 \%$ of elderly cancer patients were found to have cognitive abnormalities that needed further analysis. Impaired cognitive function was related to higher tumor stage at diagnosis, lower chance of curative treatment and significantly worse survival [13]. Another important aspect is the prediction of treatment-related decrease of cognitive function. This consideration seems to significantly influence choice of treatment. Almost $90 \%$ of seriously ill elderly cancer patients would not choose treatment if the outcome was survival with severe cognitive impairment [20]. The most commonly used cognitive state screening tool is the Mini-Mental Status Exam (MMSE).

The most important issues in the analysis of the psychological status of elderly cancer patients are depression and anxiety. A large proportion of elderly cancer patients (up to $40 \%$ ) have depression, associated with high clinical and also social impact. A large prospective study showed that depressed patients have a higher risk of postoperative complications and worse survival [12, 21]. Although 
several tools, such as the Geriatric Depression Scale, have been developed most studies have employed the psychological domain of different quality of life tests [22]. Beyond clinical relevance, depression has several social implications; thus psychological status is usually evaluated in conjunction with available social support.

CGA is time consuming and, as a result, is often neglected in clinical settings. In the last decade the new concept of frailty has been developed. The term frailty refers to the ease with which a minor stressor can have a major impact on the physical state of a vulnerable patient. It can be explained by the decrease of physiological reserves through the accumulated decline of multiple organ systems [18]. Frailty questionnaires are designed to include somatic, functional, and psychosocial domains which are suitable for quick screening to select patients for thorough analysis by CGA. This two-stepped screening method could save time as it would identify fit patients who do not require CGA. Although several validated frailty screening instruments are in use, the specificity of these tools has been rather disappointing in elderly patients with cancer.

Although several publications have reported experiences with CGA in cancer patients, there has been only a single prospective study reported on surgically treated head and neck cancer patients [23]. A recent review of this topic mainly includes CGA studies performed for sites other than head and neck [14]. Experience with CGA in the nonsurgical treatment of head and neck cancer is also limited [24]. A multicenter, prospective study is needed to clarify the value of CGA in head and neck cancer.

Finally, it should be mentioned that a multidisciplinary approach especially among surgeons, oncologists, and radiation oncologists is key to successful treatment of head and neck cancer. Multidisciplinary care in elderly should be an integrated team approach considering all relevant treatment options and develop collaboratively an individual treatment plan for each patient.

\section{References}

1. Oeppen J, Vaupel JW (2002) Demography. Broken limits to life expectancy. Science 296:1029-1031

2. Lacy PD, Piccirillo JF, Merritt MG, Zequeira MR (2000) Head and neck squamous cell carcinoma: better to be young. Otolaryngol Head Neck Surg 122:253-258

3. Janssen-Heijnen ML, Gondos A, Bray F, Hakulinen T, Brewster $\mathrm{DH}$, Brenner H, Coebergh JW (2010) Clinical relevance of conditional survival of cancer patients in Europe: age-specific analyses of 13 cancers. J Clin Oncol 28:2520-2528

4. van der Schroeff MP1, van de Schans SA, Piccirillo JF, Langeveld TP, Baatenburg de Jong RJ, Janssen-Heijnen ML (2010) Conditional relative survival in head and neck squamous cell carcinoma: permanent excess mortality risk for long-term survivors. Head Neck 32:1613-1618

5. Paleri V, Wight RG, Silver CE, Haigentz M Jr, Takes RP, Bradley PJ, Rinaldo A, Sanabria A, Bień S, Ferlito A (2010) Comorbidity in head and neck cancer: a critical appraisal and recommendations for practice. Oral Oncol 46:712-719

6. Bøje CR, Dalton SO, Primdahl H, Kristensen CA, Andersen E, Johansen J, Andersen LJ, Overgaard J (2014) Evaluation of comorbidity in 9388 head and neck cancer patients: a national cohort study from the DAHANCA database. Radiother Oncol 110:91-97

7. Bøje CR, Dalton SO, Grønborg TK, Primdahl H, Kristensen CA, Andersen E, Johansen J, Andersen LJ, Overgaard J (2013) The impact of comorbidity on outcome in 12623 Danish head and neck cancer patients: a population based study from the DAHANCA database. Acta Oncol 52:285-293

8. Gugić J, Strojan P (2013) Squamous cell carcinoma of the head and neck in the elderly. Rep Pract Oncol Radiother 18:16-25

9. Sanabria A, Carvalho AL, Vartanian JG, Magrin J, Ikeda MK, Kowalski LP (2007) Factors that influence treatment decision in older patients with resectable head and neck cancer. Laryngoscope 117:835-840

10. van der Schroeff MP, Derks W, Hordijk GJ, de Leeuw RJ (2007) The effect of age on survival and quality of life in elderly head and neck cancer patients: a long-term prospective study. Eur Arch Otorhinolaryngol 264:415-422

11. Derks W, de Leeuw RJ, Hordijk GJ (2005) Elderly patients with head and neck cancer: the influence of comorbidity on choice of therapy, complication rate, and survival. Curr Opin Otolaryngol Head Neck Surg 13:92-96

12. PACE participants, Audisio RA, Pope D, Ramesh HS, Gennari R, van Leeuwen BL, West C, Corsini G, Maffezzini M, Hoekstra HJ, Mobarak D, Bozzetti F, Colledan M, Wildiers H, Stotter A, Capewell A, Marshall E (2008) Shall we operate? Preoperative assessment in elderly cancer patients (PACE) can help. A SIOG surgical task force prospective study. Crit Rev Oncol Hematol 65:156-163

13. Extermann M, Hurria A (2007) Comprehensive geriatric assessment for older patients with cancer. J Clin Oncol 25:1824-1831

14. Shuman AG, Patel SG, Shah JP, Korc-Grodzicki B (2014) Optimizing perioperative management of geriatric patients with head and neck cancer. Head Neck 36:743-749

15. Rodin MB, Mohile SG (2007) A practical approach to geriatric assessment in oncology. J Clin Oncol 25:1936-1944

16. Huisman MG, van Leeuwen BL, Ugolini G, Montroni I, Spiliotis J, Stabilini C, de'Liguori Carino N, Farinella E, de Bock GH, Audisio RA (2014) "Timed Up \& Go": a screening tool for predicting 30-day morbidity in onco-geriatric surgical patients? A multicenter cohort study. PLoS One 9:e86863

17. Brunello A, Sandri R, Extermann M (2009) Multidimensional geriatric evaluation for older cancer patients as a clinical and research tool. Cancer Treat Rev 35:487-492

18. Hamaker ME, Jonker JM, de Rooij SE, Vos AG, Smorenburg $\mathrm{CH}$, van Munster BC (2012) Frailty screening methods for predicting outcome of a comprehensive geriatric assessment in elderly patients with cancer: a systematic review. Lancet Oncol 13:e437-e444

19. Brown T, Ross L, Jones L, Hughes B, Banks M (2014) Nutrition outcomes following implementation of validated swallowing and nutrition guidelines for patients with head and neck cancer. Support Care Cancer. doi:10.1007/s00520-014-2180-9

20. Fried TR, Bradley EH, Towle VR, Allore H (2002) Understanding the treatment preferences of seriously ill patients. N Engl J Med 346:1061-1066 
21. Passik SD, Dugan W, McDonald MV, Rosenfeld B, Theobald DE, Edgerton S (1998) Oncologists' recognition of depression in their patients with cancer. J Clin Oncol 16:1594-1600

22. Ojo B, Genden EM, Teng MS, Milbury K, Misiukiewicz KJ, Badr $\mathrm{H}$ (2012) A systematic review of head and neck cancer quality of life assessment instruments. Oral Oncol 48:923-937

23. Brugel L, Laurent M, Caillet P, Radenne A, Durand-Zaleski I, Martin M, Baron M, de Kermadec H, Bastuji-Garin S, CanouïPoitrine F, Paillaud E (2014) Impact of comprehensive geriatric assessment on survival, function, and nutritional status in elderly patients with head and neck cancer: protocol for a multicentre randomised controlled trial (EGeSOR). BMC Cancer 14:427

24. Pottel L, Lycke M, Boterberg T, Pottel H, Goethals L, Duprez F, Van Den Noortgate N, De Neve W, Rottey S, Geldhof K, Buyse V, Kargar-Samani K, Ghekiere V, Debruyne PR (2014) Serial comprehensive geriatric assessment in elderly head and neck cancer patients undergoing curative radiotherapy identifies evolution of multidimensional health problems and is indicative of quality of life. Eur J Cancer Care 23:401-412 